\title{
La bande dessinée en cours d'allemand entre comic strip et roman graphique
}

Britta Langhans, Ines Paraire et Nathalie Schnitzer

\section{(2) OpenEdition}

Édition électronique

URL : http://journals.openedition.org/trema/5055

DOI : $10.4000 /$ trema.5055

ISSN : 2107-0997

Éditeur

Faculté d'Éducation de l'université de Montpellier

Édition imprimée

ISBN : 979-10-96627-07-3

ISSN : 1167-315X

Référence électronique

Britta Langhans, Ines Paraire et Nathalie Schnitzer, « La bande dessinée en cours d'allemand entre comic strip et roman graphique », Tréma [En ligne], 51 | 2019, mis en ligne le 01 avril 2019, consulté le 06 août 2019. URL : http://journals.openedition.org/trema/5055; DOI : 10.4000/trema.5055

Ce document a été généré automatiquement le 6 août 2019

Trema 


\title{
La bande dessinée en cours d'allemand entre comic strip et roman graphique
}

\author{
Britta Langhans, Ines Paraire et Nathalie Schnitzer
}

1 Dès la fin du XIXe siècle, la littérature enfantine rencontre un franc succès outre-Rhin avec des histoires illustrées à visée moralisatrice telles que Max und Moritz (Busch, 1865) ou le livre de comptines Der Struwwelpeter (Hoffmann, 1895) ${ }^{1}$, deux œuvres ayant joué un rôle précurseur dans l'histoire de la bande dessinée et qui sont aujourd'hui considérées comme des classiques. Au milieu des années 1930, les célèbres histoires sans paroles de Vater und Sohn (Père et fils) par Erich Ohser font la joie des lecteurs du Berliner Illustrierte Zeitung dans une période par ailleurs assez sombre ${ }^{2}$. Après la seconde guerre mondiale, la bande dessinée allemande ne connait pourtant pas le même essor que la bande dessinée franco-belge et les Comics ${ }^{3}$ restent longtemps un genre mineur aux yeux de la bourgeoisie cultivée. Mais depuis les années 1990 on assiste à un véritable renouveau de la bande dessinée de langue allemande et à l'émergence d'une culture du roman graphique dans l'Allemagne réunifiée (voir notamment Rabenstein-Michel \& Benoit, 2010). Une partie importante de cette riche production a pour sujet l'enfance dans le système totalitaire de la RDA (République Démocratique Allemande), l'expérience de la chute du mur et les années qui ont suivi la réunification, autant de thématiques susceptibles d'intéresser, en France, un public de lycéens et lycéennes germanistes. Au cours des dernières décennies, cette vitalité de la production de BD de langue allemande a pu inciter les enseignants et enseignantes d'allemand à se saisir de ce médium pour en faire bénéficier leurs élèves. Une telle démarche était d'ailleurs dans l'air du temps au moment où la BD s'imposait comme outil pédagogique à part entière. Cette tendance est attestée dans le Cadre européen de référence pour les langues ${ }^{4}$ sous la rubrique «utilisation esthétique ou poétique de la langue » (CECR, 2001: 47) ; la bande dessinée trouve sa place parmi les supports recommandés, à côté notamment de la caricature, des histoires en images, ou encore des romans photos. L'exploitation de la $\mathrm{BD}$ en cours de langue soulève néanmoins des questions d'ordre méthodologique et notre contribution s'interroge, dans le prolongement d'autres travaux, sur ce que pourrait être « une démarche intégrée, qui 
articule l'enseignement de la compétence à communiquer, l'enseignement de la compétence culturelle et l'étude des particularités sémiotiques du médium, afin de mettre en valeur les singularités littéraire et plastique de l'œuvre étudiée » (Rouvière, 2012 : 286). Cette contribution s'appuie sur un travail empirique initié durant l'année universitaire 2017-2018 dans le cadre d'un séminaire de linguistique appliquée en Master $2 \mathrm{MEEF}^{5}$. Deux des auteures sont alors enseignantes d'allemand stagiaires en lycée général. Elles sont confrontées à des publics de niveau hétérogène dont la motivation pour l'apprentissage de l'allemand n'est pas manifeste. Elles mènent le travail de terrain dans les classes qui leur ont été confiées et en font le compte-rendu. La troisième participante est enseignante-chercheuse et anime ce séminaire. L'étude porte sur l'intégration de $\mathrm{BD}$ en classe de seconde, première et terminale. Il s'agit de voir si ce médium, qui semble en général apprécié des élèves ${ }^{6}$, permet de les motiver et de les familiariser à la langue et à la culture allemande par le biais de documents authentiques ${ }^{7}$. Les BD étudiées sont prioritairement des supports de l'enseignement, mais elles peuvent aussi devenir objet d'enseignement lorsque la mise en œuvre pédagogique met l'accent sur la dimension culturelle et esthétique des œuvres étudiées.

L'objectif de cette contribution est de proposer un retour d'expérience sur l'utilisation de la BD en cours d'allemand et de fournir quelques pistes pour éviter de possibles déconvenues avec ce médium. Le travail peut en effet se révéler plus complexe qu'il n'y parait, d'abord en termes de motivation pour le support : la BD allemande d'aujourd'hui est plutôt destinée à un public adulte, les adolescents sont quant à eux davantage attirés par la culture manga ${ }^{8}$. En matière d'exploitation du support, il convient par ailleurs d'éviter certains écueils, tels qu'une mauvaise appréciation des entraves culturelles à la compréhension, la sous-estimation de la complexité linguistique du support, ou encore la surestimation de la compétence des élèves en matière d'analyse de la relation texteimage.

Sur le plan méthodologique, il convient de préciser que le travail empirique dont les résultats sont exposés ici n'a pas fait l'objet en amont d'un protocole expérimental strict. Ainsi les interactions verbales entre enseignantes et élèves n'ont pas été enregistrées lors des activités menées en classe. Les échanges dialogués ne sont donc pas retranscrits mot à mot (verbatim), mais présentés dans la suite de manière synthétique.

\section{La bande dessinée en cours d'allemand : état des lieux}

La BD a acquis au fil des années une forme de légitimité éducative ${ }^{9}$ et a progressivement trouvé sa place dans les programmes officiels de l'Education nationale. L'idée de faire appel à la BD pour l'enseignement des langues en général et de l'allemand en particulier n'est donc pas nouvelle et elle a tracé son chemin éditorial depuis les années 1980. Un exemple parmi d'autres : le comic strip américain à succès Hägar the Horrible (Hägar Dünor, dit aussi Hägar le Viking) créé par Dik Browne en 1973 et traduit dans de nombreuses langues, dont la version allemande, qui fut aussi un bestseller outre-Rhin, a donné lieu à un support pédagogique largement diffusé dans les années 1980 (Kaminski, 1988) ${ }^{10}$. Parmi les publications récentes, l'ouvrage de Michaela Brinitzer (2016) ${ }^{11}$ qui fournit des idées de didactisation à partir de bandes dessinées de Thomas Körner, Simon Schwartz, Mawil et Flix mérite également d'être cité. D'autres outils pédagogiques en lien avec l'utilisation pédagogique de la $\mathrm{BD}$ ont été développés ces dernières années et sont consultables en 
ligne, notamment sur les sites Internet des Instituts Goethe de Suède (Grammatik einmal anders, 2008) et de Pologne (didactisation de plusieurs romans graphiques récents) ${ }^{12}$.

Si l'utilisation de la BD en cours de langue n'est plus aujourd'hui à proprement parler une innovation pour les didacticiens, l'abondance des outils pédagogiques qui continuent à être développés dans ce domaine montre que l'intérêt pour ce médium ne se dément pas. Sur le plan linguistique, les questions de l'acquisition, de la consolidation et la mobilisation du lexique constituent des enjeux majeurs de l'apprentissage d'une langue vivante étrangère. La BD peut être avantageusement utilisée dans ce cadre comme support d'enseignement, mais il n'est pas si simple de favoriser la construction du lexique chez les élèves sans pour autant réduire le support à une simple béquille pédagogique. On rencontre la même difficulté lorsqu'on a recours à la BD pour enseigner des faits de langue. L'un des recueils précédemment cités, Grammatik einmal anders (2008), s'efforce certes de conférer une dimension ludique au traditionnel exercice grammatical aujourd'hui décrié, mais pose tout de même la question des limites de l'instrumentalisation du support ${ }^{13}$.

6 La bande dessinée peut être aussi mise au service de la transmission de connaissances et compétences culturelles. Lorsque la narration s'inscrit dans un contexte historique particulier, elle constitue un bon levier pour l'évocation de la période en question. Le roman graphique autobiographique Drüben (De l'autre côté) de Simon Schwartz (2009) ${ }^{14}$, dans lequel l'auteur relate son enfance dans le Berlin-Est des années 1980, permet par exemple de parler de la chute du mur de Berlin en lien avec la notion de "mémoire: héritages et ruptures » en classe de seconde (cf. B.O. spécial $n^{\circ} 4$ du 29 avril 2010). En cycle terminal, la notion «lieux et formes du pouvoir» (cf. B.O. spécial $n^{\circ} 9 \mathrm{du} 30$ septembre 2010) se prête également à ce type d'approche: dans le manuel Team Deutsch pour la classe de terminale (Collectif, 2010 : 103), le célèbre roman graphique Maus de Art Spiegelman, fondé sur des entretiens entre l'auteur et son père, rescapé de la Shoah, fait l'objet d'une brève présentation dans le cadre d'une séquence consacrée à la dictature nationale socialiste et au devoir de mémoire ${ }^{15}$.

7 Des problématiques plus directement liées à l'actualité peuvent également être abordées par le biais de reportages en bande dessinée. Il existe de nombreuses ressources, notamment sur le thème de l'accueil des migrants en Allemagne et sur les peurs suscitées par l'arrivée de populations issues d'autres cultures. L'Institut-Goethe met ainsi à la disposition des enseignants et enseignantes d'allemand une exposition itinérante composée d'une série d'affiches dessinées et commentées sur le thème de la lutte contre les stéréotypes et autres clichés envers les étrangers: «Bildkorrektur - Bilder gegen Bürgerängste » (correction d'image - des images contre les peurs des citoyens) ${ }^{16}$. Le projet en ligne "Alphabet des Ankommens » (alphabet de l'arrivée) ${ }^{17}$ présente quant à lui 12 reportages en bande dessinée sur le thème de l'installation dans un pays étranger.

8 Pour une approche plus littéraire, on pourra utiliser des ressources s'appuyant sur des adaptations d'œuvres classiques ou modernes. Le Faust de Felix Görmann (2010) très librement inspiré (et transposé dans l'époque actuelle) de la pièce éponyme de Goethe, pourra constituer une porte d'entrée vers ce classique de la littérature allemande : dans son ouvrage déjà cité, Michaela Brinitzer consacre un chapitre à ce Faust revisité (Brinitzer, 2016: 78-96). Le début du XXe siècle en littérature pourra être évoqué à travers l'adaptation d'un extrait du Procès de Kafka en BD (Mairowitz \& Crumb : 1995) ${ }^{18}$, en s'appuyant notamment sur le retour d'expérience de Claire Pelosse (2006) dans une classe de terminale LV2. Enfin, l'adaptation fidèle ${ }^{19}$ par Isabel Kreitz (1996) de la nouvelle 
d'Uwe Timm (1993), Die Entdeckung der Currywurst ${ }^{20}$ pourra être exploitée pour aborder la littérature allemande de la seconde moitié du XXe siècle : un extrait de ce dernier roman graphique fait l'objet d'une didactisation assez conséquente dans le manuel Perspektiven pour la classe de 2de (Le Bourg \& Reynis, 2010 : 126-132).

\section{Compte-rendu d'expérience et pistes d'exploitation}

9 Que ce soit sur le plan littéraire, historique ou politique et citoyen, la BD a la réputation de rendre accessibles des thématiques difficiles à aborder avec des supports uniquement textuels (extraits d'œuvre littéraire, textes de presse, documents d'archives, etc.) - une hypothèse que nous avons eu l'occasion de mettre à l'épreuve dans le cadre du stage en responsabilité de deux des auteurs de cette contribution. Les deux œuvres retenues pour cette expérience s'inscrivent dans des contextes culturels et historiques en lien avec le programme de langue vivante au lycée et permettent d'aborder le thème de la famille: d'une part le roman graphique Im Himmel ist Jahrmarkt (Birgit Weyhe, 2013) ${ }^{21}$, d'autre part le comic strip Touché (Thomas Körner, 1991-...). Le premier a servi de support pour développer le thème de la famille autrefois (fin XIXe, début XXe siècle), le second celui de la famille aujourd'hui.

\section{Présentation des supports}

10 Avec Touché, Thomas Körner (alias $\odot \mathrm{TOM}$ ) s'approprie les codes de la BD humoristique dite « gros nez » (cf. Deyzieux, 2008: 60) tout en s'inscrivant dans la tradition du comic strip né aux États-Unis vers la fin du XIXe siècle et traditionnellement publié dans la presse $^{22}$. Des histoires brèves à visée généralement humoristique (d'où le terme comic) sont racontées en quelques cases alignées formant une bande (d'où le terme strip). Les strips de la série Touché paraissent chaque jour dans le quotidien berlinois taz ${ }^{23}$ depuis 1991. La série affiche donc une exceptionnelle longévité, plus de 25 ans, à raison d'une histoire par jour sauf le dimanche ${ }^{24}$. En 2000, Thomas Körner a reçu pour cette série le prix Max und Moritz ${ }^{25}$ du meilleur comic strip en langue allemande. Sans être liées à des évènements précis, les histoires de Touché, qui mettent en scène des personnages généralement anonymes dans leur vie quotidienne, sont comme une fenêtre ouverte sur la société allemande contemporaine. Si ces strips en noir et blanc ont l'avantage de pouvoir être traités dans leur intégralité, puisqu'ils présentent des histoires indépendantes les unes des autres, ils ont l'inconvénient d'être destinés à un public adulte (les lecteurs du quotidien taz), dont les préoccupations sont différentes de celles des élèves de lycée. Une présélection est donc nécessaire pour identifier les thématiques adaptées à ce public. Notre choix s'est porté sur un strip illustrant un sujet familier des élèves, la rentrée des classes.

11 Très éloignés de l'univers du comic strip et de la bande dessinée humoristique, les albums de Birgit Weyhe s'inscrivent dans la veine du roman graphique. Né aux Etats Unis à la fin des années 1970, le Graphic Novel est défini par Agnes Deyzieux comme

un récit en image débarrassé de certaines contraintes du comic book, en particulier le calibrage des pages et le découpage compartimenté des images, se rapprochant d'une conception 'romanesque' du livre de bande dessinée. L'ambition d'une pagination libre, avec un format plus proche du livre que du comic book, une division en chapitres, une libre combinaison de l'image et du texte, le choix assumé d'une esthétique du noir et blanc, une exploration des potentialités graphiques et 
narratives de la bande dessinée en sont les composantes essentielles (Deyzieux, $2008: 64)$. (2013) qui puise dans la mémoire et les archives familiales pour retracer l'histoire de ses aïeuls nés entre 1894 et 1913, à l'aide de photos, de lettres et autres témoignages ${ }^{26}$. A travers l'histoire de cette famille bourgeoise, c'est aussi un panorama de l'histoire tourmentée de l'Allemagne au XXe siècle qui s'offre au lecteur. La perspective historique peut être exploitée en cours d'allemand au-delà du travail sur la langue. Ce roman graphique se prête donc à une exploitation pédagogique qui allie les approches linguistique et culturelle. Une difficulté méthodologique réside dans l'utilisation d'extraits d'une œuvre qu'il n'est pas possible, faute de temps, de présenter dans son intégralité, ce qui est une source possible de frustration pour les élèves ${ }^{27}$. Afin que la lecture, même partielle, permette aux élèves une entrée dans l'œuvre, nous avons pris soin de sélectionner un passage présentant une unité narrative (un épisode marquant dans la vie de l'une des protagonistes) et de situer soigneusement le passage étudié dans son contexte en nous appuyant sur des éléments du prologue (p. 7-16), qui permettent notamment de comprendre que Marianne, la fillette dont il sera question ensuite, est la propre grand-mère de l'auteure.

\section{Le décodage de l'implicite dans le roman graphique de Birgit Weyhe}

L'activité a été menée en parallèle dans quatre groupes de $2^{\text {nde }}$, dont un groupe en langue vivante 1 (18 élèves) et trois groupes en langue vivante 2 (respectivement 33, 28 et 8 élèves). Selon les groupes, une à deux heures de cours y ont été consacrées. L'extrait choisi, un passage du premier chapitre (p. 23-27), a été utilisé comme illustration de la vie familiale autrefois qui s'inscrit au programme de langue vivante $2^{28}$ avec l'entrée culturelle «l'art de vivre ensemble » et la notion «mémoire : héritages et ruptures ». Le contexte historique du roman a permis de dialoguer avec les élèves autour de questions du type : comment imaginez-vous la vie d'une jeune fille à cette époque en Allemagne? En quoi se distingue-t-elle de la vôtre ? Puis l'accent a été mis sur l'événement relaté dans l'extrait choisi : à l'âge de cinq ans, Marianne fait l'expérience de la naissance d'une petite sœur. Le style graphique très soigné de B. Weyhe, à la fois épuré et précis, ainsi que la forte complémentarité entre texte et image nous semblait de nature à stimuler l'interprétation. On pouvait aussi s'attendre à ce que la compréhension de la psychologie des personnages se trouve facilitée par l'expressivité des visages.

Ces hypothèses se sont partiellement vérifiées: si la plupart des élèves n'ont pas rencontré de difficulté majeure de compréhension, l'interprétation de la relation texteimage et le décodage des éléments implicites ne sont cependant pas toujours allés de soi. Ainsi, dans le passage ci-dessous, lorsque le récitatif indique que la mère de l'héroïne se sent « indisposée » (p. 25, case 2), la grossesse de la mère n'est que suggérée et l'image ne montre pas non plus clairement qu'elle est enceinte; elle est assise dans un fauteuil et parait certes assez abattue, mais cela pourrait être tout aussi bien être dû à la fatigue, à une rage de dents, etc. 

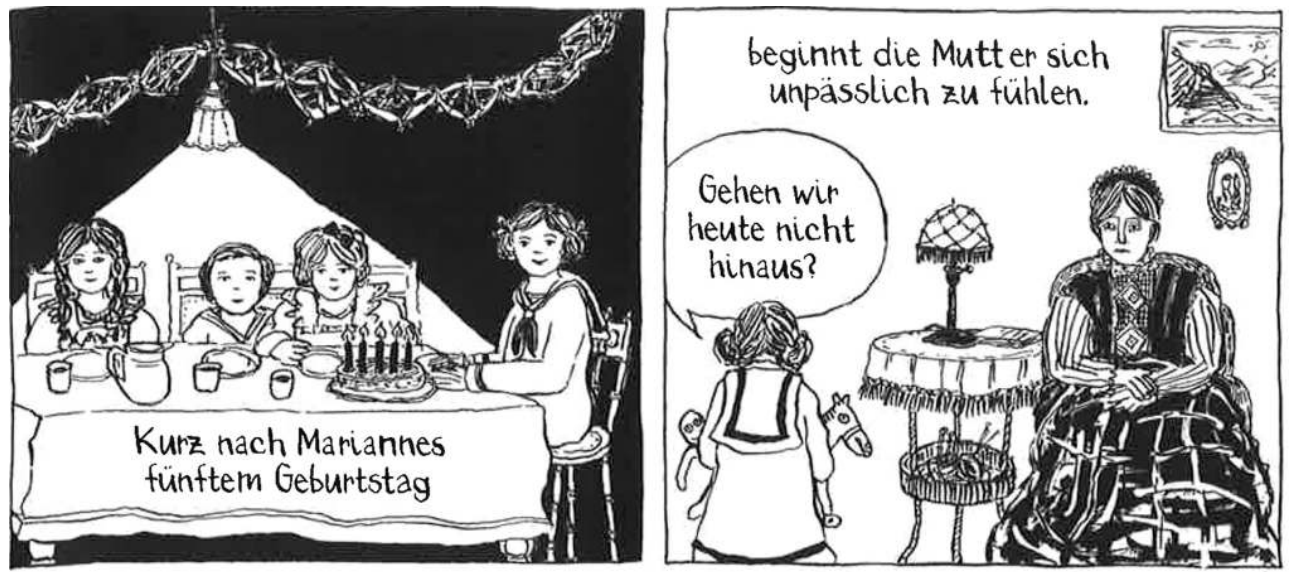

(c) avant-verlag \& Birgit Weyhe, 2013

Juste après le cinquième anniversaire de Marianne / sa mère commence à se sentir indisposée. / [Marianne :] On ne sort pas aujourd'hui ? (Weyhe, 2013 : 25)

C'est seulement à page suivante (p. 26 / cases 1 et 2), en voyant la cigogne porter un nourrisson dans son bec que le lecteur comprend pourquoi la mère était indisposée : elle attend un bébé et Marianne ne sera bientôt plus fille unique.
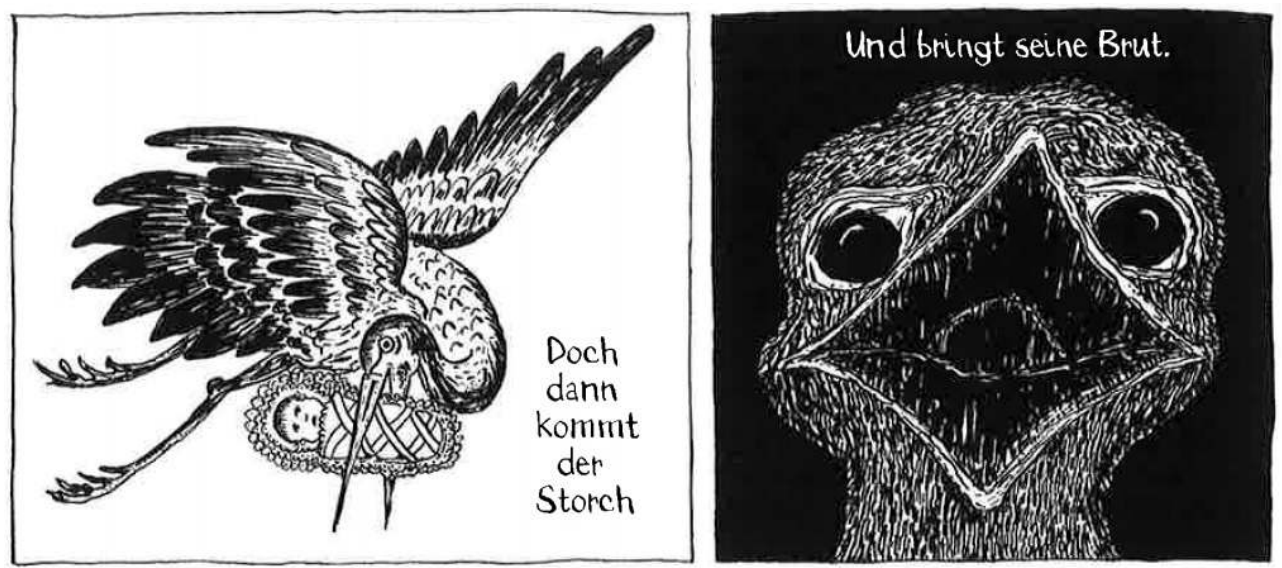

(c) avant-verlag \& Birgit Weyhe, 2013

Mais ensuite la cigogne passe / Et apporte sa couvée (Weyhe, 2013 : 26)

Dans le passage ci-dessus, l'association texte-image peut représenter un obstacle à la compréhension. Si la première image est facilement interprétable (la légende germanique de la cigogne qui apporte les enfants est connue aussi en France), la seconde en revanche l'est beaucoup moins : elle montre en gros plan un poussin au bec grand ouvert, un visuel pouvant donner lieu à d'autres interprétations que celle d'une représentation métonymique de la couvée. Si l'élève ne connait pas déjà le mot Brut, il n'a aucun moyen de savoir s'il peut s'appuyer sur l'illustration pour élucider son sens : le terme pourrait aussi bien signifier le poussin, le bec, ou encore le fait d'être affamé29. La relation texteimage est donc plus complexe qu'il n'y parait au premier abord. Comme bien souvent chez B. Weyhe, l'image est faussement redondante par rapport au texte (Cf. Schnitzer, 2017). Parfois l'image prend le relais du texte pour en préciser le sens implicite, comme dans l'exemple qui suit. Après la naissance de sa petite sœur, Marianne éprouve de la jalousie, sans que cela ne soit dit explicitement (p. 26 / case 6): 


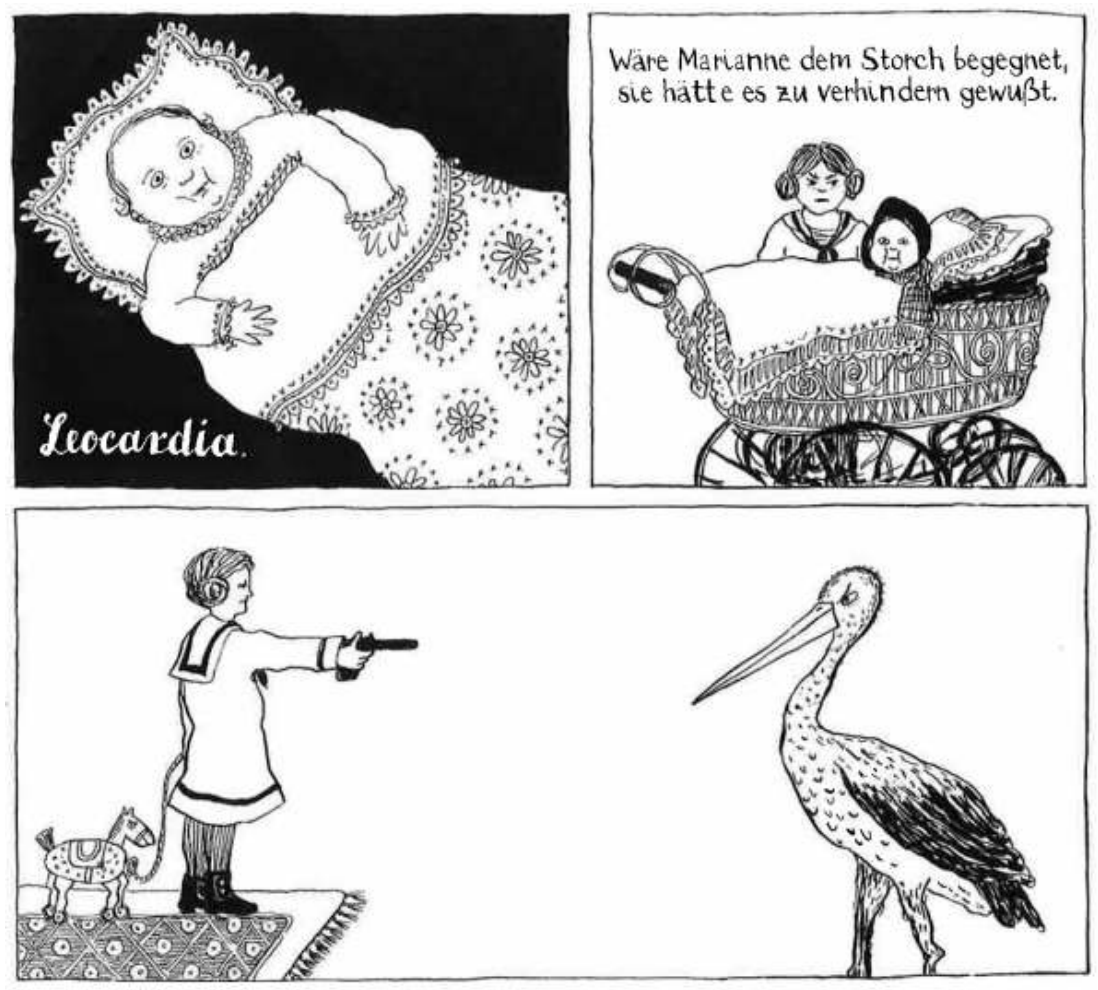

(c) avant-verlag \& Birgit Weyhe, 2013

Si Marianne avait rencontré la cigogne, elle aurait su empêcher ça. (Weyhe, 2013 : 26)

Dans la dernière case muette, on voit simplement Marianne braquer un pistolet sur la cigogne, ce qui a laissé perplexes une partie des élèves. Marianne a-t-elle tué la cigogne? Mais pourquoi? Pour comprendre que cette scène de crime n'existe que dans l'imagination de Marianne, il faut avoir préalablement bien interprété la phrase figurant dans la case précédente : l'emploi du mode de l'irréel (les formes de subjonctif 2 wäre + participe 2 et hätte + participe 2) indique qu'il s'agit d'une hypothèse non réalisée dans le passé. Ici, non seulement le texte n'exprime que de manière allusive les envies de meurtre de Marianne ("empêcher ça »), mais il est en outre décalé d'une case par rapport au visuel dévoilant la méthode radicale qui aurait permis à Marianne de demeurer fille unique.

Un peu plus loin (p. 27 / cases 1 et 2), il est question de la manière dont la petite Leocardia, surnommée Lea, est accueillie dans la famille : 

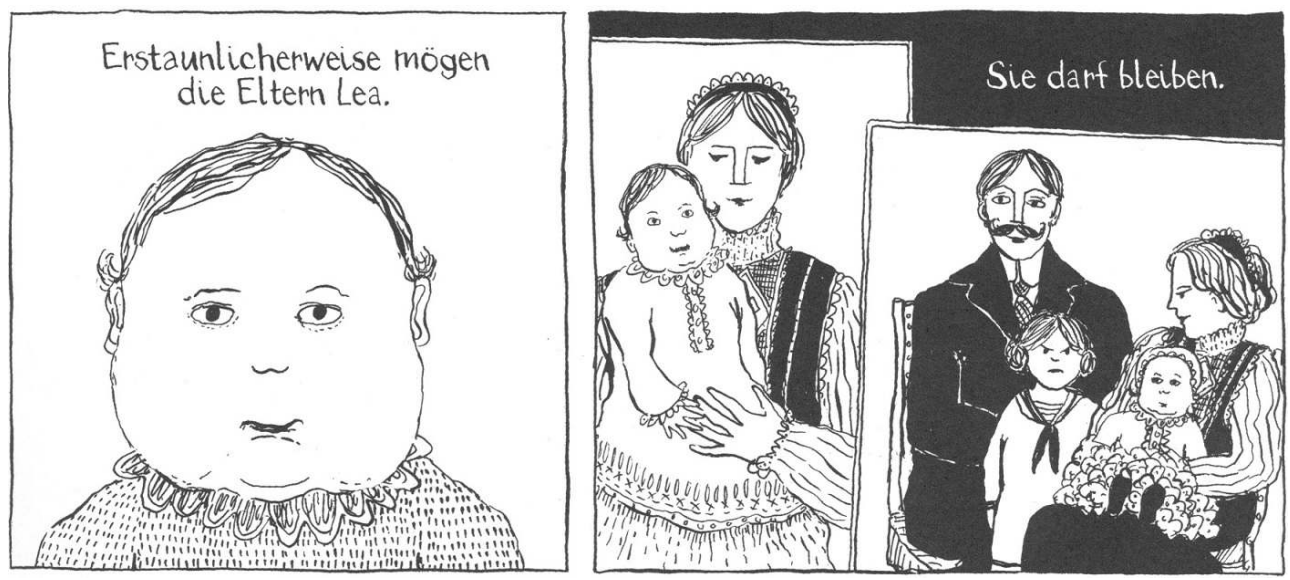

(c) avant-verlag \& Birgit Weyhe, 2013

Curieusement les parents aiment Lea / Elle a le droit de rester (Weyhe, 2013 : 27)

Quelques élèves ont eu du mal à comprendre cet étonnement naïf porté par la voix de la narratrice dont l'ironie, qui mentionne sans le reprendre à son compte le point de vue de Marianne, leur a totalement échappé. Ces subtiles variations de perspective dans le récitatif ont créé une difficulté qui n'était pas d'ordre linguistique au sens classique (lexique, morphologie, syntaxe), et qui aurait pu être mieux anticipée grâce à une observation plus attentive des personnages : sur l'image située tout à fait à droite, on distingue parfaitement la mine contrariée de Marianne qui contraste avec l'attitude protectrice de la mère portant Lea sur ses genoux. L'étonnement exprimé par le biais du modalisateur erstaunlicherweise (curieusement) ne pouvait donc que renvoyer à la jalousie de la sœur ainée.

Ces exemples montrent comment des difficultés propres au médium (décalage des contenus verbaux et iconiques) se conjuguent à des enjeux linguistiques pouvant renforcer l'incompréhension ou la mécompréhension si certaines entraves lexicales et grammaticales n'ont pas été préalablement levées.

\section{La perception de l'humour dans un comic strip de Thomas Körner}

21 Toujours dans les groupes de seconde, nous avons élaboré une autre séquence autour des relations familiales, mais cette fois-ci autour de la famille aujourd'hui avec un comic strip de Thomas Körner. Le strip choisi a servi d'introduction à la thématique des relations entre parents et enfants dans l'Allemagne d'aujourd'hui, à l'exemple des Helikoptereltern ${ }^{30}$, littéralement des "parents hélicoptères » (ou "parents poule »), qui surprotègent leurs enfants en exerçant sur eux un contrôle constant, quitte à les espionner et à harceler le personnel enseignant, depuis la crèche jusqu'au lycée. Dans l'exemple ci-dessous, on voit un couple de parents déposer leur enfant à l'école pour sa première rentrée à l'école primaire : 


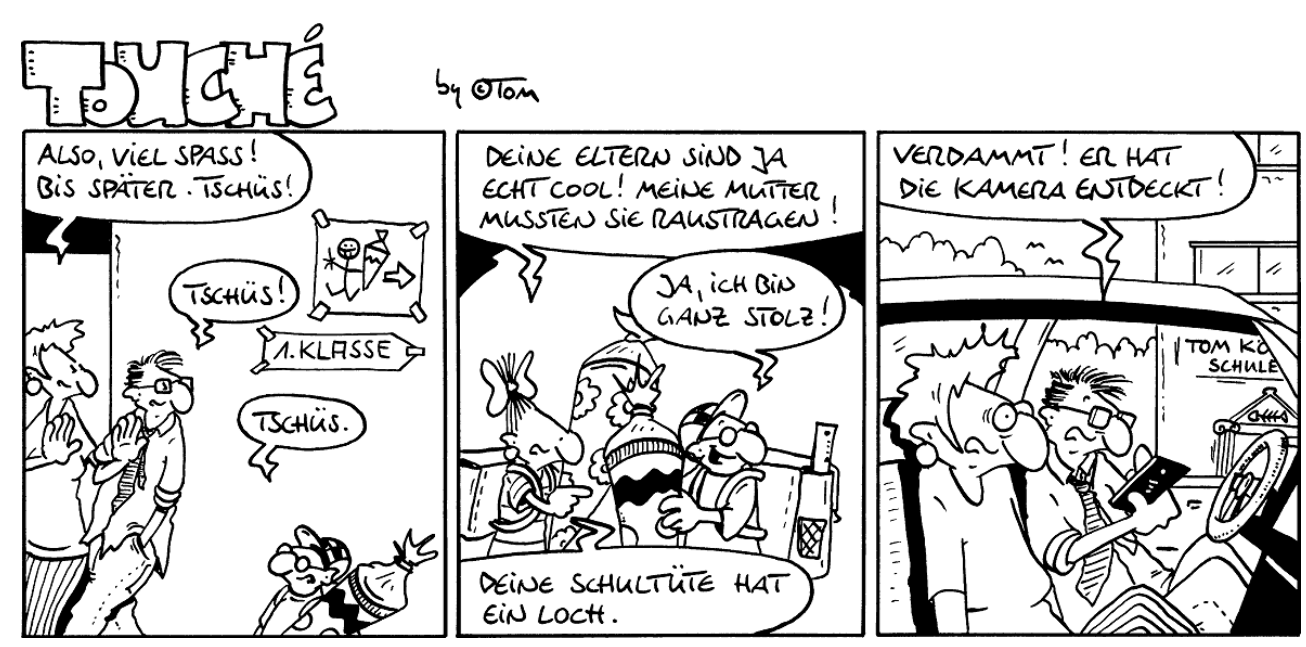

Allez, bonne journée ! A plus tard. Au revoir !/ Salut ! / Au revoir !

Tes parents sont trop cool! Ma mère, ils ont dû l'évacuer de force! / Oui, je suis très fier ! / Il y a un trou dans ta pochette.

\section{Et merde ! II a découvert la caméra! (Thomas Körner : 2016 [sans pagination])}

22 A la différence du support précédemment analysé où le récitatif était omniprésent, on remarque que le strip de Thomas Körner ne comporte que des dialogues, la question du point de vue du narrateur et d'une éventuelle superposition des instances énonciatives difficile à percevoir pour les élèves ne se pose donc pas ici. Si le volume de texte est limité par le format des bulles, le contenu implicite véhiculé par le visuel est particulièrement riche. Les implicites culturels constituent des entraves potentielles, mais aussi le caractère humoristique du support, qui est à la fois un atout et un handicap : si le ressort humoristique n'est pas perçu, alors que la forme même du comic strip crée une attente de ce type, la déception des élèves risque d'être d'autant plus grande. Dans le cas présent, le comique de la situation tient notamment à l'inversion des rôles: la décontraction des parents n'est qu'apparente et ils sont en réalité très angoissés, alors que l'enfant est tout à fait serein. La structure narrative est assez classique: elle consiste en une scène d'exposition (1e case), suivi d'un rebondissement ( $2^{\mathrm{e}}$ case), et enfin d'un dénouement, la chute de l'histoire (3e case), qui livre l'explication de l'énigme contenue dans la $2^{\mathrm{e}}$ case. La difficulté pédagogique consiste à ménager l'effet de surprise pour que le gag fonctionne ${ }^{31}$, donc à guider suffisamment les élèves, mais sans résoudre l'énigme à leur place.

Compte tenu de cette contrainte méthodologique, le strip n'a pas été présenté d'emblée dans son intégralité : les bulles ont été occultées et les élèves ont travaillé sur les trois cases projetées l'une après l'autre au tableau. Nous avons d'abord analysé les images, en particulier décodé la gestuelle et les mimiques des protagonistes pour mieux cerner leur "psychologie»: dans la case 1, les parents affichent une attitude ostensiblement détachée, alors que dans la case 3, ils semblent au contraire extrêmement tendus. Qu'estce qui explique ce changement d'attitude? L'accent a également été mis sur Schultüte, accessoire bien visible dans la case 2 , sorte de pochette surprise remplie de bonbons que les parents offrent traditionnellement aux enfants lors de la rentrée à l'école primaire (des parents qui sont accueillis dans les établissements, ce qui est rarement le cas en France). Dans une perspective interculturelle, la forte charge émotionnelle de cette journée a par ailleurs été soulignée, ainsi que la dimension très solennelle de ce qui est considéré en Allemagne comme la première rentrée, le Kindergarten ne faisant pas partie de la scolarité. Ces précisions ont permis des échanges oraux sur les expériences des 
élèves, qui n'imaginaient pas que des parents puissent être si impliqués dans la scolarisation de leur enfant ; certains avaient plutôt le souvenir de leurs propres parents les confiant sans état d'âme aux bons soins de l'Education nationale.

Les entraves culturelles ayant été levées, les élèves ont pu se concentrer sur la dimension humoristique du strip qui nécessitait une lecture fine des dialogues ${ }^{32}$ - en lien étroit avec les éléments visuels, car la relation image-texte est à double sens : si l'image, de par son caractère analogique ${ }^{33}$, est dans un premier temps de nature à faciliter la compréhension du texte, c'est ensuite le texte qui permet de désambiguïser l'image (par essence polysémique ${ }^{34}$ ), une réciprocité qui peut échapper à l'enseignant.e, pour qui l'image semble limpide - elle ne l'est en réalité que parce qu'elle entre en résonance avec le texte.

Concrètement, et afin de stimuler la motivation du groupe, l'activité n'a pas été présentée aux élèves comme un exercice de compréhension de l'allemand, mais plutôt comme un défi ludique, ceci afin que l'effort cognitif nécessaire au traitement de toutes les informations véhiculées par ce strip ne soit pas un obstacle, mais au contraire un atout pédagogique. Toujours dans le cadre du cours dialogué, l'accent a été mis sur la résolution de l'énigme de la deuxième case : le constat par la fillette de l'existence d'un trou dans la pochette surprise a intrigué les élèves qui, dans un premier temps, se sont demandé à quoi cela pouvait bien servir. Dans la troisième case, l'exclamation du père s'apercevant avec effroi que leur fils avait repéré la caméra leur a permis de lever le mystère: les élèves ont immédiumtement établi le lien entre la pochette trouée, l'existence de la caméra et le désarroi des parents... et saisi le comique de la situation : pour suivre les faits et gestes de leur fils tout au long de la journée, ils avaient caché une caméra dans sa Schultüte - d'où leur tranquillité initiale qui laisse soudain place à une réaction de panique. Cela a provoqué le rire, mais a servi également de déclencheur de parole: le champ lexical de la surveillance et du contrôle (beobachten, überwachen, spionieren) a rapidement été activé. C'est ainsi que la notion de Helikoptereltern a pu être introduite en se basant sur un exemple caricatural, certes, mais représentatif de ce type de comportement parental, phénomène sociétal prégnant en Allemagne ces dernières années dans les milieux favorisés ${ }^{35}$.

\section{Conclusion}

Le gag strip de Thomas Körner aborde un sujet familier des élèves sur un ton humoristique et décalé. La présentation de la première rentrée des classes en Allemagne sous cet angle original permet de rompre avec le traitement habituel de ce marronnier pédagogique. L'analyse de l'anecdote demande aux élèves un effort cognitif (découverte d'un fait culturel typiquement allemand ${ }^{36}$, mise en relation de l'image et du texte, décodage d'éléments implicites, travail de déduction), tout en s'appuyant sur la dimension affective dont l'importance dans l'apprentissage et la mémorisation du lexique a été soulignée par divers auteurs (voir notamment A. Boulton, 1998). Une langue s'acquiert en effet par la mobilisation de tous les sens. Cette dimension affective est également présente dans l'extrait de Im Himmel ist Jahrmarkt de Birgit Weyhe : la jalousie entre frères et sœurs est un sentiment que les élèves peuvent avoir eux-mêmes éprouvé sans forcément l'avoir verbalisé - ou même en avoir eu pleinement conscience. L'histoire de Marianne et Lea est donc susceptible de faire écho à leur propre expérience et de leur donner à réfléchir sur les relations entre frères et sœurs - à plus d'un siècle de distance. 
La familiarité des élèves avec le sujet, tout comme la présence d'une dimension affective ne sont pas pour autant une garantie de résultat. Comme toujours lorsqu'on travaille sur un support authentique, le succès de l'activité est subordonné à une préparation minutieuse pour lever toute une série d'entraves qui ne sont pas seulement culturelles et linguistiques. L'apparente facilité d'accès de la BD liée à la présence d'images susceptibles de compenser des lacunes lexicales peut, comme on l'a vu, se révéler trompeuse. En outre, le décodage des images n'est pas si intuitif qu'on pourrait le penser, mais nécessite un apprentissage, notamment pour l'interprétation des images "en séquence» (par opposition aux images uniques), qui n'est pas une faculté innée. Le développement de cette compétence de lecture des images ${ }^{37}$ chez les enfants ${ }^{38}$ et sa consolidation tout au long de la scolarité sont donc particulièrement importants dans la société actuelle dominée par les écrans, et où les sollicitations visuelles sont omniprésentes. L'enseignant.e de langue peut apporter sa pierre à l'édifice à travers l'exploitation raisonnée de bandes dessinées en langue étrangère au collège et au lycée.

\section{BIBLIOGRAPHIE}

\section{Corpus :}

Körner, T. (2016). Touché 7000, Oldenburg/Hamburg : Carlsen Verlag.

Weyhe, B. (2013a). Im Himmel ist Jahrmarkt, Berlin : Avant-Verlag.

Weyhe, B. (2013b). Kermesse au paradis (traduit de l'allemand par Élisabeth Willenz), Paris :

Cambourakis.

\section{Ouvrages cités :}

B.O. spécial $n^{\circ} 4$ (29 avril 2010). Programme d'enseignement des langues vivantes en classe de seconde générale et technologique : Ministère de l'Education nationale. http:// cache.media.education.gouv.fr/file/special_4/72/7/langues_vivantes_143727.pdf

B.O. spécial n 9 (30 septembre 2010). Programme d'enseignement de langues vivantes du cycle terminal pour les séries générales et technologiques : Ministère de l'Education nationale. http:// www.education.gouv.fr/cid53320/mene1019796a.html

Bomel-Rainelli, B. \& Demarco, A. (2011). La BD au collège depuis 1995 : entre instrumentalisation et reconnaissance d'un art. Le français aujourd'hui, Vol (172), 81-91. https://www.cairn.info/ revue-le-francais-aujourd-hui-2011-1-page-81.htm

Boulton, A. (1998). L'acquisition du lexique en langue étrangère. UPLEGESS, Vol (26), 77-87. https://hal.archives-ouvertes.fr/hal-00114355

Brinitzer, M. (2015). Zack, Deutsch lernen mit Comics. Stuttgart : Klett. 
Busch, W. (1865). Max und Moritz : eine Bubengeschichte in sieben Streichen. München : Braun \& Schneider.

CECR. Cadre européen commun de référence pour les langues : Apprendre, enseigner, évaluer (2001). Conseil de l'Europe. https://rm.coe.int/16802fc3a8

CECR. Cadre européen commun de référence pour les langues : Apprendre, enseigner, évaluer. Volume complémentaire (2018). Conseil de l'Europe. https://rm.coe.int/cecr-volumecomplementaire-avec-de-nouveaux-descripteurs/16807875d5

Collectif (2012) : Team Deutsch Lycée - Terminale. Paris : Editions Maison des langues.

De-la-Bretèque, F. (1992). Image, Lecture et Didactique. Tréma, vol. (2), 1-13. https:// journals.openedition.org/trema/2402

Demougin, F. (2002). Littérature et image : d'une lecture à l'autre. Tréma, Vol (19), 1-11. https:// journals.openedition.org/trema/1584

Deyzieux, A. (2008). Les grands courants de la bande dessinée. Le français aujourd'hui, Vol (161), 59-68. https://www.cairn.info/revue-le-francais-aujourd-hui-2008-2-page-59.htm? contenu $=$ article

Eduscol (2016). Déclinaisons culturelles Allemand, cycle 3 : Ministère de l'Education nationale. http:// cache.media.eduscol.education.fr/file/Allemand/72/6/

RA16_C3_LV_allemand_declinaison_culturelle_616726.pdf

Escande-Gauquié, P. \& Souchier, E. (2011). Matières et supports, la bande dessinée dans tous ses états, Communication \& Langages, $\mathrm{n}^{\circ}$ 167, 17-29. https://www.cairn.info/revue-communication-etlangages1-2011-1-page-17.htm

Flix (2010). Faust : der Tragödie erster Teil. Hamburg : Carlsen.

Goethe-Institut Schweden (2008). Grammatik einmal anders. http://www.goethe.de/resources/ files/pdf49/Grammatik_ueben_mit_Comics.pdf

Goethe-Institut Polen (2018). „Im Himmel ist Jahrmarkt" von Birgit Weyhe. Didaktische Materialien zum Buch. Niveau A2-B1. https://www.goethe.de/resources/files/pdf44/

final_im_himmel_ist_jahrmarkt.pdf

Greiner L. \& C. Padtberg C. (2017). Verschieben Sie die Deutscharbeit - mein Sohn hat Geburtstag : Von Helikopter-Eltern und Premium Kids. Berlin : Ullstein.

Groensteen, T. (1988). Mécanique d'un 'gag strip'. neuvième art 2.0 [sans pagination]. http:// neuviemeart.citebd.org/spip.php?article1150

Groensteen, T. (2012). Définitions. In Pascal Ory (Dir.), L'Art de la bande dessinée (pp. 17-76). Paris : Citadelles et Mazenod.

Hoffmann H. (1895). Der Struwwelpeter oder lustige Geschichten u. drollige Bilder für Kinder von 3-6 Jahren. Frankfurt am Main : Rütten \& Löning.

Kaminski, D. (1988). Hägar der Schreckliche im Kampf mit der deutschen Grammatik. Ismaning/ München : Verlag für Deutsch.

Kreitz, I. (1996). Die Entdeckung der Currywurst : nach einem Roman von Uwe Timm, Hamburg : Carlsen.

Le Bourg, S \& Reynis U. (2010). Perspektiven - Allemand 2e. Paris : Nathan.

Mairowitz, D. Z. \& Crumb R. (1995) : Kafka kurz und knapp. (traduction allemande : Ursula Grützmacher-Tabori). Frankfurt a. M. : Zweitausendeins. 
Mawil (2013). Wir können ja Freunde bleiben. Berlin : Reprodukt.

Missiou, M. \& Anagnostopoulou, D (2010). La bande dessinée -œuvre littéraire en classe de français langue étrangère : étude de cas en Grèce. 11emes Rencontres des Chercheurs en Didactique des Littératures. Genève. https://www.researchgate.net/publication/285393177_La_bande_dessinee_oeuvre_litteraire_en_classe_de_francais_langue_etrangere_etude_de_cas_en_Grece Pelosse, C. (2006). Le procès de Kafka en BD. Les Langues Modernes, Vol (4), 16-19.

Rabenstein-Michel, I. \& Benoit M. (éd.) (2010). « Krack! Tschock ! Pflatsch ! Bummmm ! ». La BD de langue allemande (à suivre...). Germanica, Vol (47). https://journals.openedition.org/ germanica/1078

Rabouin, Claudine (2007). Le Procès de Kafka en BD. In P. Zard, Sillage de Kafka (pp. 511-524). Paris : Le Manuscrit.

Rouvière, N. (éd.) (2012). Bande dessinée et enseignement des humanités. Grenoble, ELLUG.

Schnitzer, N. (2017). La relation texte-image dans le roman graphique Kermesse au paradis de Birgit Weyhe. Studia Philologia, Vol (3), 85-98. http://www.studia.ubbcluj.ro/arhiva/ abstract_en.php?editie=PHILOLOGIA\&nr=3\&an=2017\&id_art=15461

Schwartz, S. (2009). Drüben! Berlin : Avant-Verlag.

Schwartz, S. (2011). De l'autre côté, traduction Élise Lacharme. Paris : Sarbacane.

Stella-Guyot, D. (2007). Réaliser une bande dessinée. CRDP de Poitou-Charentes : Dupuis.

Timm, U. (1993). Die Entdeckung der Currywurst. Köln : Kiepenheuer \& Witsch.

Timm, U. (1996). La Découverte de la saucisse au curry, traduction Bernard Kreiss. Paris : Seuil.

Vincent Gerard, A. \& Vayssettes, B. (2018). Les jeunes adultes et la lecture. Le Centre national du livre, Ipsos. https://www.centrenationaldulivre.fr/fr/ressources/etudes_rapports_et_chiffres/

\section{NOTES}

1. Max und Moritz comme le Struwwelpeter (Pierre l'ébouriffé) sont aujourd'hui des héros représentatifs de l'ère culturelle germanophone dont l'évocation "permet d'entrer dans un monde virtuel qui fait rêver et constitue un vecteur de motivation appréciable » (Eduscol 2016 : 10). Dernière consultation pour tous les liens de cette contribution : 14/06/2018.

2. Plus connu sous le pseudonyme de E.O. Plauen, Erich Ohser publie la série humoristique Vater und Sohn dans la Berliner Illustrierte Zeitung entre 1934 et 1937. Hostile au régime nazi, il est finalement arrêté en 1944 et se suicidera en prison.

3. Terme utilisé en allemand pour désigner la bande dessinée.

4. Publié par le Conseil de L'Europe en 2001, il définit des niveaux en langue étrangère (de A1 à

C2) dans différents domaines de compétence. Ce cadre fait aujourd'hui référence en Europe et audelà. Un volume complémentaire a été publié récemment (CECR, 2018).

5. Métiers de l'Enseignement, de l'Education et de la Formation.

6. Selon une récente étude réalisée par Ipsos pour le Centre national du livre, les 15-25 ans sont 54\% à lire des «livres illustrés » tous genres confondus (Vincent Gerard \& Vayssettes, 2018: 44). Selon la même étude, les $15-25$ ans sont aussi $57 \%$ à lire des romans.

7. Documents non conçus à l'origine pour un cours de langue.

8. Selon l'étude Ipsos citée ci-dessus, sur les $54 \%$ de lecteurs de «livres illustrés ", plus de la moitié (33\%) lisent des mangas. 
9. L'expression peut s'entendre aussi au sens du développement de formations professionnelles dédiées à ce médium (cf. Escande-Gauquié \& Souchier, $2011: 17$ ).

10. Ce livret est conçu pour être utilisé par l'enseignant comme support d'appoint en complément du manuel scolaire. Il est composé de 18 chapitres ayant tous pour point de départ un strip du fameux Wiking. Chaque chapitre est associé à des objectifs communicationnels permettant d'activer (ou de réactiver) des compétences grammaticales.

11. L'ouvrage reproduit des extraits assez longs des albums sélectionnés (jusqu'à une douzaine de pages) et fournit des informations sur leur auteur et l'arrière-plan civilisationnel, ainsi que des aides lexicales et un panel d'exercices pour guider l'analyse des documents.

12. Documents PDF à télécharger gratuitement.

13. Exemple au chapitre 2 (p.10-12) : deux planches extraites du roman graphique d'Isabel Kreitz (1996) sont présentées comme support pour un point de révision sur « les verbes » sans que l'on comprenne ce qui a motivé ce choix. Par ailleurs, les bulles étant vidées de leur contenu, l'exercice proposé porte sur beaucoup plus que ce qui est annoncé en ouverture: il s'agit de réécrire l'extrait en commençant chaque phrase par un mot imposé, un pronom personnel sujet dans la plupart des cas, ce qui est d'une part artificiel en termes de structuration des énoncés allemands, et d'autre part sans rapport avec l'extrait original (dans lequel une seule phrase s'ouvre sur un pronom personnel sujet).

14. Ce roman graphique existe en version française (Schwartz, 2011).

15. Publié en 1986 (t.1) et 1991 (t.2) en anglais, l'ouvrage est ici utilisé dans sa version allemande.

16. https://www.goethe.de/ins/fr/fr/spr/unt/ver/aus/21128140.html

17. http://alphabetdesankommens.de/

18. Il s'agit de la traduction allemande d'un ouvrage paru en anglais (Mairowitz \& Crumb : 1993).

19. L'adjectif « fidèle » n'est associé ici à aucun jugement de valeur, la fidélité au texte littéraire n'étant pas en soi un gage de qualité : voir à ce propos les remarques critiques de J. Baetens (2009: [en ligne, sans pagination] \$2-3) sur une vision ancienne de l'adaptation qui considère l'œuvre source comme « un modèle indépassable ».

20. Titre français : La Découverte de la saucisse au curry (Timm, 1996).

21. Cette œuvre qui a été traduite en français, l'année même de sa parution, sous le titre Kermesse au paradis (2013b).

22. L'un des inventeurs du comic strip est le dessinateur américain Rudolph Dirks (1877-1968). Fils d'immigrés allemands, il s'inspire des aventures de Max und Moritz pour créer The Katzenjammer Kids. La série est publiée à partir de 1897 dans un supplément dominical du New York Journal. Quelques-unes de ces histoires sont accessibles sur le site de la bibliothèque numérique de la Ohio State University. A titre d'exemple, le strip daté du 20 août 1899 et intitulé "The Katzenjammer Kids lose their clothes " met en scène la maman allemande des deux garnements; la présence de phylactères est une nouveauté pour l'époque: https:// library.osu.edu/dc/concern/generic_works/g732z867d

23. taz est l'acronyme de tageszeitung (littéralement "quotidien»). Ce journal politiquement orienté à gauche a son siège à Berlin, mais sa diffusion est nationale.

24. Tous les strips de la série Touché sont réunis dans des albums composés chacun de 500 histoires. Le dernier album en date, le 7500, est paru en 2018.

25. Prix décerné tous les deux ans (depuis 1984) à l'occasion du festival international de la bande dessinée d'Erlangen (Bavière) par un jury composé de professionnels. https://www.comicsalon.de/de/max-und-moritz-preis-seit-1984

26. Les deux premiers chapitres sont consacrés à ses grand-mères Marianne et Herta, le troisième au grand-oncle Karl-Friedrich, les deux derniers à ses grands-pères Edgar et Eduard.

27. Une lecture suivie de l'œuvre, dont une partie se serait déroulée en autonomie à la maison, était difficilement envisageable avec des élèves d'un niveau de langue inférieur ou égal à B1. Une lecture intégrale en classe répartie sur le trimestre n'était pas non plus possible dans les 
conditions de notre stage en responsabilité - ce qui ne signifie en rien qu'une telle activité ne puisse être menée avec succès dans un contexte différent (Cf. Missiou, M. \& Anagnostopoulou, 2010).

28. «La classe de seconde se consacre à l'art de vivre ensemble, dans le présent, le passé, et l'avenir, fondé sur différentes formes de sociabilité ou de solidarité, qu'il s'agisse de l'évolution des sociétés traditionnelles ou de la redéfinition des rapports sociaux, partagés entre valeurs collectives et individualisme " (BO spécial $\mathrm{n}^{\circ} 4,29$ avril 2010: 5). http:// cache.media.education.gouv.fr/file/special_4/72/7/langues_vivantes_143727.pdf

29. Ici la difficulté avait été anticipée, l'enseignante ayant pris soin d'indiquer le sens du mot Brut (vocabulaire de réception). D'autres entraves lexicales (sich unpässlich fühlen : être indisposée, der Storch : la cigogne, erstaunlicherweise : curieusement) ont dû également être levées.

30. De l'anglais helicopter parents. L'expression est apparue aux Etats-Unis dans les années 1990 pour désigner ce phénomène de société.

31. On peut rattacher la série Touché au genre du " gag strip » ou " gag-a-day strip », bien décrit par Thierry Groensteen (1988) dans son article « Mécanique d'un 'gag strip' ».

32. Afin de lever les entraves lexicales, les traductions de raustragen (littéralement: porter à l'extérieur), stolz (fier) et verdammt! (et merde!) ont été indiquées en marge des cases projetées au tableau.

33. L'image (photographie ou dessin figuratif) ressemble à l'objet qu'elle représente, alors que le mot ne ressemble pas à son référent extralinguistique ; il est réputé «arbitraire » au sens de Ferdinand de Saussure.

34. Dans la case 2 , sans le texte associé, l'observateur ne peut pas savoir que la pochette est trouée; il pourrait en revanche imaginer que le garçon et sa camarade sont en train d'échanger leurs pochettes - ce qui n'est absolument pas le cas.

35. Le phénomène a donné lieu à de nombreuses publications. Voir par exemple cette enquête réalisée par deux journalistes du très sérieux magazine Der Spiegel, L. Greiner \& C. Padtberg (2017), dont le titre est évocateur : Verschieben Sie die Deutscharbeit - mein Sohn hat Geburtstag: Von Helikopter-Eltern und Premium Kids (Reportez le contrôle d'allemand - c'est l'anniversaire de mon fils : à propos des parents hélicoptères et des enfants "premium»). Ce phénomène fait le bonheur des éditeurs qui publient des livres-conseil pour éviter de devenir un parent hélicoptère ou qui dénoncent les effets néfastes d'une telle éducation sur le développement de l'enfant.

36. Une partie des élèves connaissait déjà le rituel de la Schultüte, ayant déjà traité cette thématique au collège. Le phénomène des Helikoptereltern leur était en revanche tout à fait inconnu.

37. L'expression "lecture des images », que nous employons par commodité, est controversée pour des raisons qu'il n'est pas possible de développer ici (cf. Demougin, 2002).

38. Cf. F. De-la-Bretèque (1992: 10) : «C'est seulement aux environs de six ans que l'enfant est capable de mettre de l'ordre dans un récit en images (et réciproquement de comprendre la relation de temps établie dans une séquence)».

\section{RÉSUMÉS}

Le recours à la bande dessinée en cours de langue n'est plus aujourd'hui une nouveauté, mais l'utilisation de ce médium à des fins didactiques demeure un exercice complexe, car les entraves 
à la compréhension sont souvent plus nombreuses qu'il n'y parait au premier abord. Cet article fait le bilan d'une expérience menée dans des classes d'allemand de lycée par deux enseignantes stagiaires à partir de deux supports représentatifs du renouveau actuel de bande dessinée outreRhin.

While the presence of comics in foreign language classes is no longer a novelty, using this medium for didactic purposes remains a delicate exercise, as students face many challenges in making sense of them. This paper reviews the experiment led in a French high school by two trainee German teachers who worked with two books representing the current renewal of comics in Germany.

\section{INDEX}

Mots-clés : didactique des langues, bande dessinée, comic strip, roman graphique, allemand Keywords : foreign language didactics, comics, comic strip, graphic novel, German

\section{AUTEURS}

BRITTA LANGHANS

ESPE Aix-Marseille

\section{INES PARAIRE}

ESPE Aix-Marseille

\section{NATHALIE SCHNITZER}

Aix-Marseille-Université, Echanges EA4236 